\title{
Glucagon-like peptide-1 (GLP-1) receptor agonists, obesity and psoriasis: diabetes meets dermatology
}

\author{
D. J. Drucker • C. F. Rosen
}

Received: 13 June 2011 / Accepted: 29 July 2011 /Published online: 3 September 2011

(C) Springer-Verlag 2011

\begin{abstract}
Type 2 diabetes mellitus is characterised by beta cell failure, which frequently develops in the setting of insulin resistance. Inflammation contributes to the pathophysiology of type 2 diabetes by impairing insulin action in peripheral tissues and via reduction of beta cell function. Inflammation may also play an important role in the development of complications that arise in patients with type 2 diabetes. Hence, the antiinflammatory actions of commonly used glucoselowering drugs may contribute, indirectly, to their mechanisms of action and therapeutic benefit. Herein we highlight the anti-inflammatory actions of glucagonlike peptide-1 (GLP-1), which exerts direct and indirect actions on immune function. The observations that GLP-1 receptor agonists exert anti-inflammatory actions in preclinical studies, taken together with case reports linking improvements in psoriasis with GLP-1 receptor agonist therapy, illustrates the emerging clinical implications of non-classical anti-inflammatory actions of incretin-based therapeutics.
\end{abstract}

Keywords Beta cell - Dipeptidyl peptidase-4 - GIP - GLP-1 Immune $\cdot$ Incretin $\cdot$ Inflammation $\cdot$ Skin $\cdot \mathrm{T}$ cell

\section{J. Drucker $(\bowtie)$}

Department of Medicine, Samuel Lunenfeld Research Institute, Mount Sinai Hospital, University of Toronto, 600 University Ave TCP5-1004, Toronto, ON M5G 1X5, Canada e-mail: drucker@lunenfeld.ca

\section{F. Rosen}

University Health Network, University of Toronto,

Toronto, ON, Canada

\author{
Abbreviations \\ DPP-4 Dipeptidyl peptidase-4 \\ GLP-1 Glucagon-like peptide-1 \\ iNKT Invariant natural killer T \\ PASI Psoriasis Area and Severity Index \\ Th T helper
}

Psoriasis is a common skin disorder characterised by chronic inflammation, varying degrees of scaling, erythema, itching and plaque formation. Psoriasis is widely viewed as an immune-mediated inflammatory disease that is associated with the expansion and activation of $\mathrm{T}$ helper (Th)1, Th17 and Th22 cells, and the increased expression and levels of cytokines detectable locally in skin lesions and, frequently, systemically in the circulation [1]. Patients with psoriasis exhibit increased rates of cardiovascular disease, obesity and type 2 diabetes - conditions characterised by enhanced local and/or systemic inflammation. However, little evidence supports a common genetic or mechanistic basis for shared causality amongst these diverse disorders.

In this issue of Diabetologia, Hogan and colleagues report an improvement of psoriasis in three obese patients with type 2 diabetes several weeks after the initiation of therapy with the glucagon-like peptide-1 (GLP-1) receptor (GLP-1R) agonists exenatide and liraglutide [2]. The improvement in psoriasis was modest but clinically appreciable, with reduced itching in the index case, and a reduction in Psoriasis Area and Severity Index (PASI) scores, from 15.3 to $10.2,13.2$ to 10.8 , and 4.8 and 3.8 , respectively. Analysis of the number of circulating invariant natural killer T (iNKT) cells, a known subset of CD1ddependent NKT cells that express an invariant $\mathrm{T}$ cell 
receptor $\alpha$ chain, revealed that, in response to liraglutide therapy, there was an increase in the number of NKT cells in the circulation and a corresponding reduction in the number of iNKT cells in psoriatic plaques. GLP1R mRNA transcripts were detectable in iNKT cell lines generated from healthy human donors, and liraglutide robustly increased cAMP accumulation in resting iNKT cells, although this activation was attenuated in cells activated with anti-CD3. Although GLP-1 increased cytokine production in resting iNKT cells, both GLP-1 and liraglutide inhibited cytokine secretion following treatment with phorbol myristate acetate/ionomycin, or in iNKT cells activated by co-culture with glycolipidtreated CD1d-transfected C1R cells, actions blocked by the GLP-1R antagonist exendin (9-39). Hence, the authors infer that the putative immunoregulatory actions of GLP-1 on iNKT cells may have contributed to the improvement in clinical psoriasis observed in the three patients [2].

Although originally identified as a beta cell G-proteincoupled receptor linked to the glucose-dependent stimulation of insulin secretion (Fig. 1), GLP-1Rs have subsequently been localised to the central and peripheral nervous system, lung, kidney, gastrointestinal tract, heart and blood vessels [3], where they mediate a diverse number of actions distinct from control of glucose homeostasis [4]. More recent studies have demonstrated widespread Glp1r expression in numerous immune cell subpopulations, including thymoyctes, splenocytes, bone marrow-derived cells, and regulatory $\mathrm{T}$ cells [5]. Consistent with the results reported by Hogan et al., GLP-1R agonists have previously been shown to increase cAMP formation in multiple lymphocyte populations in a GLP-1R- and exendin (9-39)-dependent manner [5]. Furthermore, several reports have described disease remission and enhanced numbers of regulatory $\mathrm{T}$ cells in experimental murine models of autoimmune type 1 diabetes treated with GLP-1R agonists in the absence of concomitant therapy with immunomodulatory agents $[6,7]$. GLP-1R activation also modestly reduced stromal cell-derived factor-1-enhanced lymphocyte migration in human $\mathrm{CD}^{+}$lymphocytes [8], whereas genetic disruption of Glp1r produced detectable defects in thymocyte and lymphocyte proliferation following stimulation ex vivo [5]. Hence, the available evidence supports a role for the GLP-1R in controlling lymphocyte proliferation and/or migration in murine and human immune cells.

Evidence from both preclinical and clinical studies supports the view that obesity and type 2 diabetes are frequently associated with increased local and systemic inflammation. The thiazolidinedione class of glucoselowering agents, exemplified by pioglitazone and rosiglitazone, exerts pleiotropic anti-inflammatory effects in multiple peripheral tissues, including the cardiovascular system [9]. Moreover, thiazolidinediones exert anti-
Fig. 1 The classical (green arrows) and emerging (blue arrow) metabolic actions of GLP-1. The dotted line indicates an indirect action

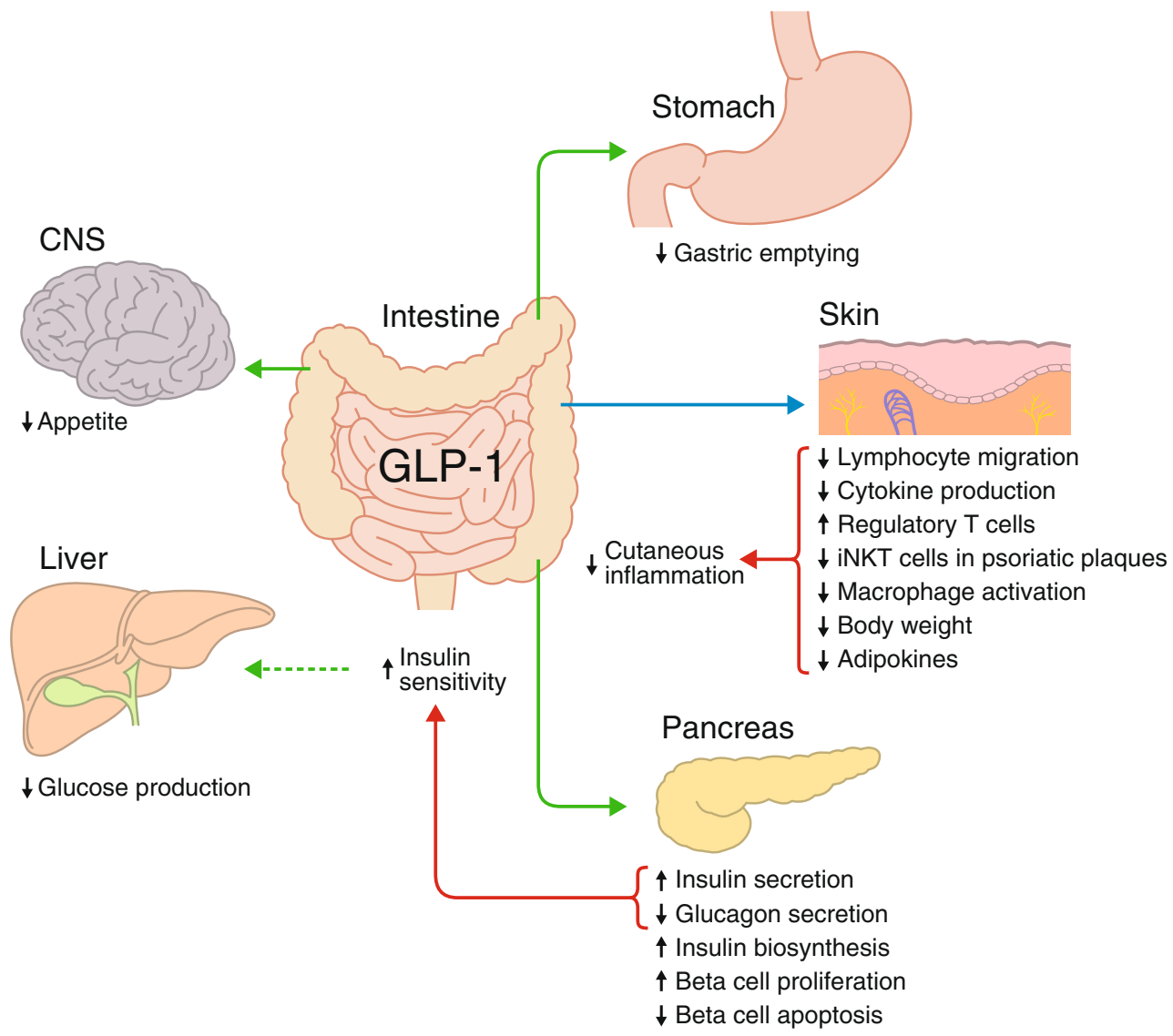


proliferative actions in the skin and enhance keratinocyte differentiation, and several pilot studies have explored the utility of these agents in the treatment of psoriasis [10]. An activated immune system in obese diabetic individuals may be associated with tissue infiltration of proinflammatory macrophages and increased cytokine levels, reduction of insulin action/and or impairment of beta cell function [11]. Indeed, therapeutic agents that specifically target inflammatory pathways have shown promise for the treatment of type 2 diabetes in humans. Intriguingly, some glucose-lowering agents may produce anti-inflammatory actions independent of changes in glucose control. Pioglitazone has been shown to exert an anti-psoriatic effect when co-administered with the synthetic retinoid acitretin to non-obese non-diabetic participants with moderate to severe plaque-type psoriasis in a 12 week randomised clinical trial [12]. In the patients reported by Hogan et al., the improvement in psoriasis was relatively rapid following the initiation of treatment with exenatide or liraglutide, with no significant improvement in glycaemic control noted [2]. Although the reductions in PASI score were modest, they occurred rapidly, and psoriasis does not usually improve spontaneously without therapy. It is therefore tempting to add GLP-1R agonists to the small list of glucose-lowering agents that exert anti-inflammatory actions independent of changes in glucoregulation.

Might there be an alternative explanation for the improvement in psoriasis following initiation of therapy with GLP-1R agonists? Glp1r and Gcg mRNA transcripts have been detected in RNA isolated from mouse skin and, using immunocytochemistry, the GLP-1R protein has been localised to regions of the epidermis adjacent to hair follicles [13]. A GLP-1R-immunoreactive protein was also detected by western blotting using extracts from cultured mouse skin cells and GLP-1 enhanced ERK1/2 phosphorylation in skin cells in vitro. Whether a functional GLP-1R is also expressed in normal or psoriatic human skin cells is at present unknown; however, GLPIR mRNA transcripts can be detected in expressed sequence tags from human skin (GenBank accession number BG675444.1). Hence, the putative biology of the cutaneous GLP-1-GLP-1R system clearly requires further clarification.

The epidemiological linkage of psoriasis with obesity, and the actions of GLP-1R agonists to reduce food intake and produce weight loss, merit consideration. In the Nurses' Health Study II [14], increases in BMI were linked to a progressive increase in the risk of developing psoriasis. In addition, weight gain and higher waist circumference, hip circumference and WHR were associated with an increased incidence of psoriasis [14]. Obesity is more common in patients with difficult to treat psoriasis, and a higher BMI may affect the response to anti-psoriatic therapy [15]. Intriguingly, observational studies demon- strate that patients with a higher BMI may have more severe psoriasis, and weight loss has been associated with improvements in PASI scores in patients treated with biological agents; the underlying mechanisms are incompletely understood [16]. Similarly, several case reports describe the improvement and near resolution of psoriasis following weight loss achieved through intestinal bypass surgery [15]. In a 24 week randomised trial, the consequences of moderate weight loss were examined in obese patients with moderate to severe chronic plaque psoriasis treated with ciclosporin. A mean reduction in body weight of $7 \%$ in participants following a low-energy diet resulted in significantly more patients responding (according to reduction in PASI score) to low-dose ciclosporin [17].

Although Hogan et al. describe a rapid subjective improvement in psoriasis symptoms prior to the achievement of significant weight loss in their patients [2], the actions of GLP-1R agonists to reduce energy intake and produce body weight loss and the relative contribution of these effects to the overall objective improvement in skin lesions and improvements in PASI scores after 6 weeks is difficult to ascertain. A small observational study reported that a brief period of fasting alone improved the signs and symptoms of psoriasis [18], and a 4 week low-energy diet improved the clinical severity of psoriasis independent of changes in weight loss [19]. GLP-1R agonists are known to produce a rapid induction of anorexia, and the two patients prospectively treated with liraglutide experienced weight loss of 5.1 and $6.2 \mathrm{~kg}$ after only 6 weeks of therapy. As even short interventions of hypoenergetic diets that produce moderate weight loss can also result in significant reductions in tissue and systemic inflammation, attribution of the precise GLP-1R-dependent mechanisms leading to the improvement of psoriasis in these patients remains challenging. Hence, determination of whether GLP-1R agonists can reduce psoriasis disease activity independent of changes in energy intake or weight loss is an important question for future investigation.

The extrapancreatic actions of incretin-based therapies are broad and expanding (Fig. 1), but have not classically included the skin or immune system as key target organs. Dipeptidyl peptidase-4 (DPP-4), also known as CD26, is widely expressed in the epidermis and on multiple immune cell subtypes, and preclinical studies demonstrating cutaneous vasculitis in non-human primates with some, but not all, DPP-4 inhibitors [20], has heightened awareness of the potential consequences of DPP-4 inhibition on the skin. To date, few cutaneous allergic reactions have been reported with DPP-4 inhibitors, although a recent report of druginduced bullous pemphigoid in patients treated with vildagliptin $(n=4)$ and sitagliptin $(n=1)$ requires further investigation [21]. Similarly, although patients treated with exenatide or liraglutide may experience injection-site 
reactions and, rarely, localised transient urticaria, the skin has not traditionally been viewed as a site of incretin action. The findings of improved psoriasis and functional GLP-1Rs on iNKT cells coupled to a reduction of cytokine secretion open up a new area of investigation for incretin biology, and re-emphasise the role of clinical acumen in advancing our understanding of GLP-1 biology in humans.

Contribution statement DJD and CFR reviewed the literature, drafted and wrote the manuscript and approved the final version without any outside assistance

Duality of interest D.J. Drucker has served as an advisor or consultant within the past 12 months to Amylin Pharmaceuticals, Arisaph Pharmaceuticals, Diartis Pharmaceuticals, Eli Lilly, GlaxoSmithKline, Merck Research Laboratories, Novo Nordisk, NPS Pharmaceuticals, Takeda and Transition Pharmaceuticals. Neither D.J. Drucker nor his family members hold stock directly or indirectly in any of these companies. C.F. Rosen has no relevant duality of interest to disclose.

\section{References}

1. Davidovici BB, Sattar N, Prinz JC et al (2010) Psoriasis and systemic inflammatory diseases: potential mechanistic links between skin disease and co-morbid conditions. J Invest Dermatol 130:1785-1796

2. Hogan AE, Tobin AM, Ahern T et al (2011) Glucagon-like peptide-1 (GLP-1) and the regulation of human invariant natural killer $\mathrm{T}$ cells-lessons from obesity, diabetes and psoriasis. Diabetologia. doi:10.1007/s00125-011-2232-3

3. Bullock BP, Heller RS, Habener JF (1996) Tissue distribution of messenger ribonucleic acid encoding the rat glucagon-like peptide 1 receptor. Endocrinology 137:2968-2978

4. Drucker DJ (2006) The biology of incretin hormones. Cell Metab 3:153-165

5. Hadjiyanni I, Siminovitch KA, Danska JS, Drucker DJ (2010) Glucagon-like peptide-1 receptor signalling selectively regulates murine lymphocyte proliferation and maintenance of peripheral regulatory T cells. Diabetologia 53:730-740

6. Hadjiyanni I, Baggio LL, Poussier P, Drucker DJ (2008) Exendin4 modulates diabetes onset in nonobese diabetic mice. Endocrinology 149:1338-1349

7. Zhang J, Tokui Y, Yamagata $\mathrm{K}$ et al (2007) Continuous stimulation of human glucagon-like peptide-1 (7-36) amide in a mouse model
(NOD) delays onset of autoimmune type 1 diabetes. Diabetologia 50:1900-1909

8. Marx N, Burgmaier M, Heinz P et al (2010) Glucagon-like peptide-1(1-37) inhibits chemokine-induced migration of human CD4-positive lymphocytes. Cell Mol Life Sci 67:3549-3555

9. Libby P, Plutzky J (2007) Inflammation in diabetes mellitus: role of peroxisome proliferator-activated receptor- $\alpha$ and peroxisome proliferator-activated receptor- $\gamma$ agonists. Am J Cardiol 99:27B$40 \mathrm{~B}$

10. Sertznig P, Seifert M, Tilgen W, Reichrath J (2008) Peroxisome proliferator-activated receptors (PPARs) and the human skin: importance of PPARs in skin physiology and dermatologic diseases. Am J Clin Dermatol 9:15-31

11. Donath MY, Shoelson SE (2011) Type 2 diabetes as an inflammatory disease. Nat Rev Immunol 11:98-107

12. Mittal R, Malhotra S, Pandhi P, Kaur I, Dogra S (2009) Efficacy and safety of combination acitretin and pioglitazone therapy in patients with moderate to severe chronic plaque-type psoriasis: a randomized, double-blind, placebo-controlled clinical trial. Arch Dermatol 145:387-393

13. List JF, He H, Habener JF (2006) Glucagon-like peptide-1 receptor and proglucagon expression in mouse skin. Regul Pept 134:149-157

14. Setty AR, Curhan G, Choi HK (2007) Obesity, waist circumference, weight change, and the risk of psoriasis in women: Nurses' Health Study II. Arch Intern Med 167:1670-1675

15. Bremmer S, van Voorhees AS, Hsu S et al (2010) Obesity and psoriasis: from the Medical Board of the National Psoriasis Foundation. J Am Acad Dermatol 63:1058-1069

16. Bardazzi F, Balestri R, Baldi E, Antonucci A, de Tommaso S, Patrizi A (2010) Correlation between BMI and PASI in patients affected by moderate to severe psoriasis undergoing biological therapy. Dermatol Ther 23(suppl 1):S14-19

17. Gisondi P, del Giglio M, Di Francesco V, Zamboni M, Girolomoni G (2008) Weight loss improves the response of obese patients with moderate-to-severe chronic plaque psoriasis to low-dose cyclosporine therapy: a randomized, controlled, investigator-blinded clinical trial. Am J Clin Nutr 88:1242-1247

18. Lithell H, Bruce A, Gustafsson IB et al (1983) A fasting and vegetarian diet treatment trial on chronic inflammatory disorders. Acta Derm Venereol 63:397-403

19. Rucevic I, Perl A, Barisic-Drusko V, Adam-Perl M (2003) The role of the low energy diet in psoriasis vulgaris treatment. Coll Antropol 27(suppl 1):41-48

20. Drucker DJ (2007) Dipeptidyl peptidase-4 inhibition and the treatment of type 2 diabetes: preclinical biology and mechanisms of action. Diabetes Care 30:1335-1343

21. Skandalis K, Spirova M, Gaitanis G, Tsartsarakis A, Bassukas I (2011) Drug-induced bullous pemphigoid in diabetes mellitus patients receiving dipeptidyl peptidase-IV inhibitors plus metformin. J Eur Acad Dermatol Venereol. doi:10.1111/j.1468-3083.2011.04062.x 